while only $2.6 \%$ of the patients felt worse in autumn, in comparison of $10.3 \%$ in spring and $6.0 \%$ in summer. $24.4 \%$ of the patients felt relieved in summer, while surprising, only $2.7 \%$ felt better in spring, with a lowest rate in the four season. However, $48.1 \%$ of the patients believed there were no seasonal differences. Conclusions: More patients had an onset of AS in summer, compared to other seasons. More patients felt worse in winter and better in summer. Nearly half of AS patients considered that there were no seasonal differences in the deterioration or improvement of the symptoms.

References:

[1] Karmacharya P, Pathak R, Aryal MR, Giri S, Donato AA (2016) Seasonal variation in acute gouty arthritis: data from Nationwide Inpatient Sample. Clin Rheumatol 35: 523-525.

[2] Feldthusen C, Grimby-Ekman A, Forsblad-d'Elia H, Jacobsson L, Mannerkorpi $\mathrm{K}$ (2016) Seasonal variations in fatigue in persons with rheumatoid arthritis: a longitudinal study. BMC Musculoskelet Disord 17: 59.

Disclosure of Interest: Y. Jiang: None declared, M. Yang: None declared, Z. Lin: None declared, Q. Wei: None declared, S. Cao: None declared, J. Gu Grant/research support from: Guangzhou Science and Technology Plan Projects [grant number 2006Z2-E0221] and 5010 Subject of Sun Yat-sen University (2009-2010)

DOI: 10.1136/annrheumdis-2017-eular.4167

\title{
SAT0432 CORRELATION BETWEEN THE SPINAL MRI FINDINGS AND NEW BONE FORMATION FACTOR (DKK-1)I N PATIENTS WITH SPONDYLOARTHRITIS
}

\section{Z. Zhao, G. Wang, Y.Y. Wang, J.S. Yang, J. Zhu, F. Huang. Rheumatology,} Chinese PLA General Hospital, Beijing, China

Background: Recent prospective data suggest that spinal inflammatory damage in patients with ankylosing spondylitis will eventually convert into fat. In these complex inflammatory lesions, bone formation and inflammation are not synchronized. The molecular basis responsible for new bone formation in SpA patients is still unclear. Serum level of dickkopf-1 (Dkk-1), the natural inhibitor of WNT protein, is a main factor in limiting new bone formation.

Objectives: In this study, we aimed to assess the correlation between the secreted protein Dkk-1 and abnormal findings on spinal MRI through a prospective study of SpA.

Methods: Thirty patients with active axial $\mathrm{SpA}(\mathrm{axSpA})$ who fulfilled the ASAS axSpA criteria were enrolled. All patients received an injection of recombinant human TNF receptor-antibody fusion protein (YISAIPU) at a dosage of 50 $\mathrm{mg} / \mathrm{week}$ for 6 months. Patient report outcome measure questionnaires and physical examination, blood tests were completed according to the study protocol. All patients were scored for bone marrow edema and fat infiltration on spinal MRI imaging. The spinal MRI imaging of the patients before and after the treatment were blindly reviewed and scored using the SPARCC scoring system by two individuals who were familiar with the system.

Results: There are 28 male and two female patients (mean age: $31 \pm 5.5 \mathrm{yrs}$, range: $22-41$; mean duration: $93.5 \pm 75.8$; HLA-B27(+): $96.7 \%$ (29/30)). In patients who finished the 6 month anti-TNF treatment, the ESR, CRP, BASDAI, BASFI, BASMI and ASDAS-CRP were significantly decreased $(P<0.01)$. Serum Dkk-1 concentration was also significantly decreased $(P<0.05)$, as were the edema measurements of spinal bone marrow $(P<0.05)$, but not with the before and

Table 1. Clinical indexes, serum DKK-1 and spine imaging scores before and after treatment

\begin{tabular}{lcc}
\hline & Before treatment & After treatment \\
\hline ESR $(\mathrm{mm} / \mathrm{h})$ & $23.78 \pm 22.27$ & $5.03 \pm 4.63^{\star *}$ \\
CRP $(\mathrm{mg} / \mathrm{dl})$ & $2.59 \pm 2.90$ & $0.40 \pm 0.52^{\star *}$ \\
BASDIA & $6.23 \pm 1.29$ & $2.52 \pm 1.84^{\star \star}$ \\
BASFI & $5.78 \pm 1.44$ & $2.69 \pm 1.72^{\star \star}$ \\
BASMI & $2.46 \pm 1.91$ & $0.69 \pm 1.21^{\star \star}$ \\
ASDAS-CRP & $3.77 \pm 0.83$ & $1.58 \pm 0.74^{\star \star}$ \\
DKK-1 $(\mathrm{ng} / \mathrm{ml})$ & $98.23 \pm 113.41$ & $51.88 \pm 41.90^{\star}$ \\
Spine-BME & $20.27 \pm 23.53$ & $6.08 \pm 8.09^{\star \star}$ \\
Spine- FAT & $10.08 \pm 10.38$ & $13.81 \pm 15.34$ \\
${ }^{*} \mathrm{p}<0.05 ;{ }^{* \star} \mathrm{p}<0.01$ & &
\end{tabular}

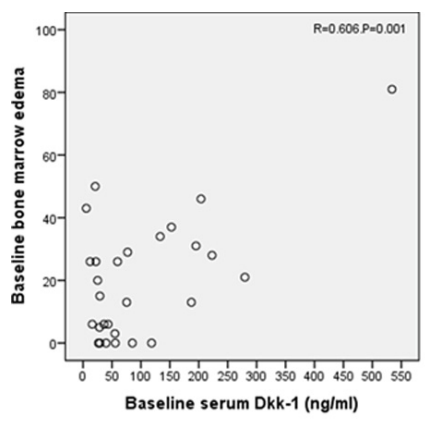

Figure 1. Serum Dkk-1 level is correlated with bone marrow edema at baseline

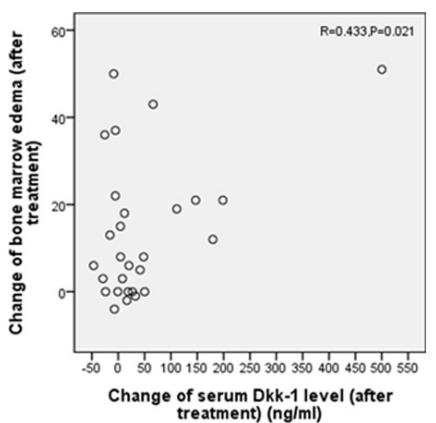
treatment) (ng/ml)

Figure 2. After treatment, the change of serum Dkk-1 level is correlated with change of bone marrow edema after treatment differences in fat infiltration scores $(p>0.05)$. (Table1). Correlation analysis found that serum Dkk-1 concentration before treatment was significantly correlated with spinal bone marrow edema scores $(P<0.01)$. The differences in serum Dkk-1 levels significantly correlate with differences in spinal MRI bone marrow edema scores after treatment $(P<0.05)$.(Figure 1 and 2$)$.

Conclusions: Spinal marrow edema may have a role in predicting new bone formation in the spine, since the change of serum Dkk-1 level is correlated with change of spinal marrow edema. And Dkk-1 may participate in the molecular basis of the TNF inhibitor's blockade of new bone formation. Further research is needed on patients who have received long-term TNF antagonist treatment to find the time points when serum Dkk-1 level reaches a stabilized plateau. Increased knowledge in this area will be helpful when assessing a predictive marker for the timing of treatment withdrawal.

Disclosure of Interest: None declared

DOI: 10.1136/annrheumdis-2017-eular.3160

\section{SATURDAY, 17 JUNE 2017}

\section{Psoriatic arthritis}

SAT0433 ANTI-TNF TREATMENT IN RHEUMATOID ARTHRITIS AND ANKYLOSING SPONDYLITIS PATIENTS IS ASSOCIATED WITH A STRONG INCREASE OF PALMOPLANTAR PUSTULOSIS BUT NOT OF PSORIASIS VULGARIS

J. Ruwaard ${ }^{1}$, E. van der Vlugt ${ }^{1}$, M. Nurmohamed ${ }^{1}$, T. Rispens ${ }^{2}$,

G.J. Wolbink ${ }^{1,2} .{ }^{1}$ Amsterdam Rheumatology and immunology Center / Reade;

${ }^{2}$ Department of Immunopathology, Sanquin Research and Landsteiner

Laboratory Academic Medical Centre, Amsterdam, Netherlands

Background: The prevalence of paradoxical psoriasis developed with biological use is already studied. However, none of these studies discriminate between psoriasis vulgaris (PV) and palmoplantar pustulosis (PPP), while these might be different entities (1). The prevalence in general population is $2-4 \%$ for $P V$ and $0.01-0.05 \%$ for PPP $(1-3)$. Moreover, most reports in the literature imply only a role for anti-Tumor necrosis factors (anti-TNF), although, a few cases described paradoxical psoriasis in patients treated with biologicals other than anti-TNF.

Objectives: To study the prevalence and incidence density of paradoxical psoriasis and palmoplantar pustulosis in patients with rheumatoid arthritis (RA) and ankylosing spondylitis (AS) treated with biological therapy. Second to investigate differences between paradoxical psoriasis and palmoplantar pustulosis.

Methods: Data were collected from the observational cohorts of AS and RA patients. 1499 consecutive patients were included for calculating prevalence and incidence density. Incidence density is calculated per 1000 person years. For calculating differences, only biological naïve patients $(n=830)$ were included. Kaplan Meier curve was used to show the difference in time in onset.

Results: In all, 13 cases of PPP and 16 cases of PV were observed in both the RA as AS cohorts. In AS patients 1.73\% developed PPP and $1.38 \%$ PV. In RA patients respectively $0.66 \%$ and $0.99 \%$.

The incidence density of PPP in RA was $2.1(95 \% \mathrm{Cl} 0.7-3.6)$, for PV $3.2(95 \% \mathrm{Cl}$ 1.4-5.0). In AS, 4.7 (95\% Cl 0.6-8.8) for PPP and 3.7 (95\% Cl 2.3-12.7) for PV. Although not statistically significant, PPP was more prevalent in adalimumab (0.94\%) compared to etanercept $(0.34 \%)$. In contrast, PV occurs in $0.53 \%$ in adalimumab and $0.92 \%$ in etanercept treated patients. PPP was only observed in anti-TNF, PV was also observed in 1 patient treated with tocilizumab and 1 with abatacept. A difference was observed in the time to event, with a median of 6 months (IQR 4-16 months) for PPP and 50 months (IQR 11-67 months) for PV; $p=0.003$ (figure 1). Discontinuation of biological treatment was indicated in $80 \%$ of the PPP patients and $18.2 \%$ PV patients.

Figure 1. Difference between patients who developed psoriasis vulgaris versus palmoplantar pustulosis on biological therapy.
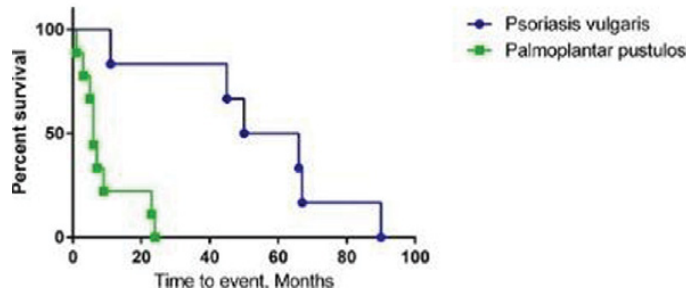

Conclusions: Our findings show that biological therapy in patients with RA or AS is associated with a 13 to 35 fold increase in prevalence of PPP. While the prevalence of biological-associated PV is lower than the prevalence of PV in the general population. In this study PV and PPP are different from each other regarding prevalence, time to onset and consequences for biological treatment, and therefore should be considered as separate entities.

\section{References:}

[1] J Dermatolog Treat 2011 Apr:22(2):102-5.

[2] Lancet 2015 Sep 5;386(9997):983-94.

[3] Acta Derm Venereol 1971;51(4):284-8. 
Disclosure of Interest: None declared

DOI: 10.1136/annrheumdis-2017-eular.3758

\section{SAT0434 NETWORK META-ANALYSIS ON THE EFFICACY OF NOVEL THERAPEUTIC AGENTS IN PATIENTS WITH PSORIATIC ARTHRITIS}

T.T. Cheung, M.F. Tsoi, Y. Fei, C.S. Lau, B.M.Y. Cheung. Medicine, The University of Hong Kong, Hong Kong, Hong Kong

Background: Novel therapeutic agents are more effective than DMARDs in the management of psoriatic arthritis. However, direct comparisons of efficacy between these novel therapeutic agents are lacking.

Objectives: This network meta-analysis aims to compare the relative efficacies between different novel therapeutic agents.

Methods: Literature searching was conducted in MEDLINE, EMBASE, Scopus, ISI Web of Science, Cochrane Library, Clinicaltrials.gov and recent rheumatology conference abstracts up to Nov 2016. 2 independent researchers analysed the articles. For inclusion, randomised, placebo-controlled trials must report the proportion of patients achieving ACR20, ACR50, ACR70 and PASI75 responses. The outcomes of this network meta-analysis were the proportion of patients achieving ACR20, ACR50, ACR70 and PASI75 responses with reference to placebo and etanercept.

Results were analysed using random effect model by $R$ statistics (version 3.3.1) with statistical package netmeta (version $0.9-2$ ). The heterogeneity of the study results was determined by the $\mathrm{I}^{2}$ statistics.

Results: 18 trials were included into this study. In general, all novel therapeutic agents demonstrated superior efficacies than placebo. With reference to etanercept, apremilast and ustekinumab were associated with less proportions of patients achieving ACR20 response (odds ratio [95\% confidence interval]: 20mg apremilast: 0.18 [0.07-0.48]; 30mg apremilast: 0.24 [0.09-0.62]; 45mg ustekinumab: 0.26 [0.09-0.73]; 90mg ustekinumab: 0.32 [0.11-0.90]).

Etanercept was not different from apremilast, ustekinumab, golimumab, adalimumab, ixekizumab, certolizumab, ixekizumab and secukinumab in terms of ACR20 and ACR50 responses.

Golimumab and infliximab were associated with greater proportions of patients achieving PASI75 response, though the difference did not reach statistical significance. (odds ratio [95\% confidence interval]: golimumab: 3.51 [0.44-28.2]; infliximab: 5.97 [0.89-40.2]).

Table 1. Network meta-analysis on proportion of patients achieved ACR20 and PASI75 response with reference to etanercept

\begin{tabular}{lcc}
\hline & ACR20 response & PASI75 response \\
\hline 10mg Tofacitinib BID & $0.38[0.12 ; 1.22]$ & $0.68[0.13 ; 3.54]$ \\
150mg Secukinumab & $0.64[0.22 ; 1.90]$ & $0.49[0.07 ; 3.19]$ \\
20mg Apremilast BID & $0.18[0.07 ; 0.49]$ & $0.39[0.08 ; 1.83]$ \\
300mg Secukinumab & $0.77[0.21 ; 2.80]$ & $0.67[0.10 ; 4.56]$ \\
30mg Apremilast BID & $0.24[0.09 ; 0.63]$ & $0.48[0.10 ; 2.22]$ \\
45mg Ustekinumab & $0.26[0.09 ; 0.75]$ & $1.18[0.15 ; 9.09]$ \\
5mg Tofacitinib BID & $0.25[0.08 ; 0.81]$ & $0.64[0.12 ; 3.29]$ \\
80mg Ixekizumab Q2w & $0.40[0.12 ; 1.27]$ & $2.74[0.49 ; 15.37]$ \\
80mg Ixekizumab Q4w & $0.35[0.11 ; 1.11]$ & $1.73[0.32 ; 9.31]$ \\
90mg Ustekinumab & $0.32[0.11 ; 0.92]$ & $1.40[0.18 ; 10.74]$ \\
Adalimumab & $0.33[0.13 ; 0.86]$ & $0.77[0.17 ; 3.50]$ \\
Certolizumab & $0.42[0.12 ; 1.39]$ & $\mathrm{NA}$ \\
Etanercept (reference) & 1.00 & 1.00 \\
Golimumab & $0.93[0.25 ; 3.40]$ & $3.51[0.44 ; 28.22]$ \\
Infliximab & $0.74[0.26 ; 2.12]$ & $5.97[0.89 ; 40.22]$ \\
Placebo & $0.10[0.04 ; 0.22]$ & $0.09[0.03 ; 0.35]$ \\
\hline NA: not & &
\end{tabular}

NA: not applicable.

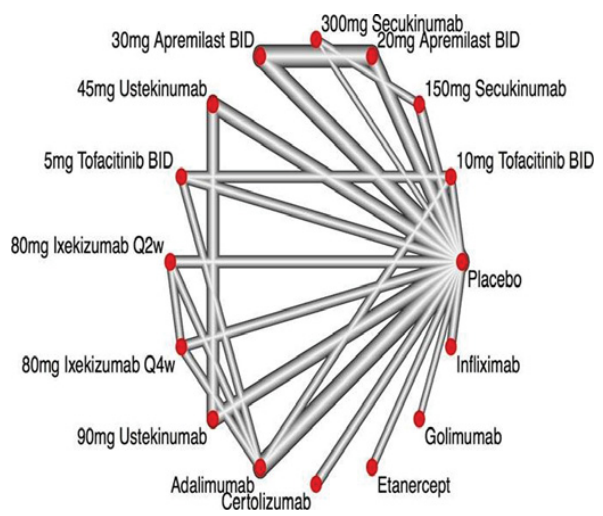

Conclusions: Apremilast and ustekinumab were less efficacious than etanercept in terms of ACR20 response. All the novel therapeutic agents demonstrated comparable efficacies in terms of ACR50, ACR70 and PASI75 responses.

Disclosure of Interest: None declared

DOI: 10.1136/annrheumdis-2017-eular.5096

\section{SAT0435 IL17 CORRELATES POSITIVELY WITH TGF-BETA 1 AND DKK1 AND INVERSELY WITH BMP2 AND 4 IN SYNOVIAL} MEMBRANE OF PATIENTS WITH PSORIATIC ARTHRITIS

J. Pinto-Tasende, M. Fernandez-Moreno, M.E. Vazquez-Mosquera, J.C. Fernandez-Lopez, N. Oreiro-Villar, M. Bejerano-Herrería, B. Acasuso-Pardo de Vera, F.J. Blanco-García. INIBIC-Rheumatology, Complejo Hospitalario Universitario A Coruña, A Coruña, Spain

Background: Immune and non-immune cells contribute to the pathology of chronic arthritis and they can contribute to tissue remodeling and repair as well as disease pathogenesis. An important role for their products such as TGF-beta 1, IL17 or BMPs has been suggested in homeostatic and remodeling mechanisms in arthritis. BMP signaling could have an anti-inflammatory role in the control and maintenance of low levels of pro-inflammatory factors in healthy joints or the early stage of RA.

Objectives: to analyze and compare serum levels, gene expression and immunohistochemistry (IHC) in synovial membrane of inflammation and bone destruction/regeneration biomarkers in patients with psoriatic arthritis (PsA), undetermined seronegative arthritis (USA), Osteoarthritis of the knee (kOA) and ankylosing spondylitis (AS).

Methods: We recruited 45 consecutive patients with chronic knee arthritis referred for undergoing arthroscopies (17 PsA, 12 USA, 12 kOA, 4 AS). Synovial membrane was processed for IHC analysis and quantification of mRNA expression ratio by qRT-PCR. Serum levels of TGF-beta 1, IL6, IL17 and IL22, DKK1, Sclerostin, BMP2, BMP4, Wnt1 and Wnt5a were measured (ELISA). We analyzed and compared these data with the demographic, clinical, analytical and radiological characteristics of the patients. Data were analyzed using the SPSS version 17.0 software and statistical significance was defined as $\mathrm{P}<0.05$.

Results: We obtained valid synovial membrane samples from 41 patients for $\mathrm{IHC}$, RNA extraction and purification from 29 patients for analyze mRNA expression and serum from 38 patients for protein levels measurement. IL17 gene expression was higher in PsA patients $(p=0.027)$ and correlated positively with DKK1 $(r=0.424$, $\mathrm{p}=0.022)$ and negatively with BMP2 $(\mathrm{r}=-0.396, \mathrm{p}=0.033)$ and BMP4 $(\mathrm{r}=-0.472$, $\mathrm{p}=0.010$ ). IHC reactivity for TGF-beta 1 in synovial tissue was higher in patients with psoriatic arthritis ( $p$ 0.010) and correlated positively with IL17 ( $r=0.389$, $p=0.012)$ and DKK1 $(r=0.388, p=0.012)$. Moreover, serum levels of TGF-beta 1 were significantly increased in PsA with erosions $(p=0.044)$.

Conclusions: IL17 gene expression in synovial membrane from patients with psoriatic arthritis was higher than in seronegative undetermined arthritis, osteoarthritis and ankylosing spondylitis patients, correlating positively with DKK1 and negatively with bone morphogenetic proteins 2 and 4 . In addition, TGF-beta in synovial tissue, necessary for the activation of Th17 cells, was higher in patients with psoriatic arthritis, in relation to IL17 and DKK1 increased. Serum TGF beta 1 levels were also higher in patients with erosive disease.

References:

[1] Varas A, Valencia J, Lavocat F, Martínez VG, Thiam NN, Hidalgo L, et al. Blockade of bone morphogenetic protein signaling potentiates the proinflammatory phenotype induced by interleukin-17 and tumor necrosis factor- $\alpha$ combination in rheumatoid synoviocytes. Arthritis Res Ther. 2015;17:192. doi: 10.1186/s13075-015-0710-6.

Acknowledgements: This work has been supported by grant Pl11/00390 from Plan Nacional de Investigación Científica, Desarrollo e Innovación Tecnológica 2008-2011 y cofinanciado por el ISCIII-Subdirección General de Evaluación y Fomento de la Investigación - Fondo Europeo de Desarrollo Regional (FEDER).

Disclosure of Interest: None declared

DOI: 10.1136/annrheumdis-2017-eular.4427

\section{SAT0436 DURABILITY OF APREMILAST RESPONSE IN PATIENTS WITH PSORIATIC ARTHRITIS: LONG-TERM (208-WEEK) RESULTS} FROM THE PALACE 1 TRIAL

A. Kavanaugh ${ }^{1}$, D.D. Gladman ${ }^{2}$, J.J. Gomez-Reino ${ }^{3}$, S. Hall ${ }^{4}$,

E. Lespessailles ${ }^{5}$, P.J. Mease ${ }^{6}$, G. Schett ${ }^{7}$, M. Mcllraith ${ }^{8}$, N. Delev ${ }^{8}$, M. Paris ${ }^{8}$ L. Teng ${ }^{8}$, J. Wollenhaupt ${ }^{9} .{ }^{1}$ UCSD School of Medicine, la Jolla, United States; ${ }^{2}$ Toronto Western Research Institute, Toronto, Canada: ${ }^{3}$ Hospital Clínico Universitario, Santiago, Spain; ${ }^{4}$ Monash University, CabriniHealth, Melbourne, Australia; ${ }^{5}$ University of Orléans, Orléans, France; ${ }^{6}$ Swedish Medical Center and University of Washington School of Medicine, Seattle, United States; ${ }^{7}$ University of Erlangen-Nuremberg, Erlangen, Germany; ${ }^{8}$ Celgene Corporation, Summit, United States; ${ }^{9}$ Schön Klinik Hamburg Eilbek, Hamburg, Germany

Background: Optimizing treatment choice in psoriatic arthritis (PsA) necessitates an understanding of the long-term effects of therapies across varied manifestations of this complex disease. Data from 4 years of apremilast (APR) treatment in PALACE 1 were used to examine disease control across markers of active inflammation, such as SJC, as well as improvements in patient (pt) functionality, as assessed using the HAQ-DI.

Objectives: Evaluate long-term outcomes with APR treatment after $\geq 1$ DMARD or biologic in pts with active PsA.

Methods: Pts were randomized $(1: 1: 1)$ to placebo (PBO), APR $30 \mathrm{mg}$ BID (APR30), or APR $20 \mathrm{mg}$ BID (APR20). The PBO-controlled phase continued to Wk 24, at which time all remaining PBO pts were re-randomized to APR30 or 